\title{
Predictive Factor to Castrate-Resistant Prostate Cancer (CRPC) after Primary Androgen Deprivation Therapy (ADT): Single Center Experience in Indonesia
}

\author{
Taufiq Nur Budaya, Horie Shigeo
}

\begin{abstract}
ADT is the main therapy in prostate cancer, especially in advanced stages, although ADT does not limit the progression of this disease to become CRPC, where the mortality rate will be much higher when CRPC has occurred. This study aims to examine corellating factors influenced the short duration to CRPC after primary ADT administration. 205 prostate cancer patients with CRPC or $M C R P C$ in Saiful Anwar Malang Hospital from 2013 to 2018 were included in this study. Data recorded were age, initial PSA level, Gleason score, prostate cancer stage, type of ADT, nadir PSA, time between ADT and nadir PSA, and testosterone levels after ADT. To see the independent factors that influence the occurrence of CRPC, the Cox proportional hazards regression model was used. The average age of patients was $67,53 \pm 6.86$ years with an average level of initial PSA of $674.87 \pm 1405.8080$ / dL. The average time for CRPC to occur was $24.7 \pm 9.74$ months. In multivariate analysis it was found that the stage of cancer with metastasis (HR 1,616, p 0,048), testosterone level after ADT was > 20ng / dL (HR 4,638, p 0,000), nadir PSA > 4ng / dL (HR 1,716, p 0,023) and time to reach Nadir PSA $<6$ months (HR 1.596, p 0.004) is a significant independent factor for CRPC occurrence. The conclusion is cancer stage, testosterone levels after ADT, nadir PSA and time needed to reach nadir PSA were independent factors for CRPC in patients with prostate cancer who had primary $A D T$.
\end{abstract}

\section{Keywords: ADT, CRPC, Nadir PSA, Prostate Cancer}

\section{INTRODUCTION}

Prostate cancer is the most common cancer in men, in 2012 an estimated 1,1 million cases in the worldwide, $15 \%$ were diagnosed with cancers. The frequency of autopsied prostate cancer is approximately equal in the whole world (1). The prevalence of prostate cancer at $<30$ years of age is $5 \%$, then increases with an odds ratio of 1.7 per decade, to a prevalence of 59\% (48-71\%) at age> 79 years (2).

The incidence of prostate cancer varies throughout the world, the highest spread throughout Australia / New Zealand and North America where the incidence reached 111.6 percent of the population of 100,000 , while in western Europe and the north the incidence was 94.9 and 85 in

Revised Manuscript Received on January 2, 2020.

* Correspondence Author

Taufiq Nur Budaya*, Urology Department, Medical Faculty of Universitas Brawijaya/Saiful Anwar Hospital Malang, Indonesia, Email: taufiq_fkub03@yahoo.com

Horie Shigeo, Urology Department, Juntendo University, Tokyo, Japan
100,000 inhabitants. Low incidence rates was in the southeastern part of 4.5-10.5 per 100,000 inhabitants (1)

In three education center hospitals at Jakarta, Surabaya and Bandung, the number of patients with prostate cancer in for the past 8 years was 1,102 patients with an average age of 67.18 years. The most common stage of the disease when coming to treatment is an advanced stage with $59.3 \%$ of cases (3). With a pattern of prostate cancer patients, where nearly $60 \%$ of patients come in advanced conditions, treatment with androgen deprivation therapy (ADT) is the main standard therapy of prostate cancer in Indonesia (3).

Although primary ADT has been given, the journey of prostate cancer will continue to become Castrate Resistant Prostate Cancer (CRPC), or at the CRPC stage with metastases (mCRPC), where at this stage there were progression of prostate cancer characterized by an increase in prostate specific antigen (PSA) or the presence of metastases in other organs even though testosterone levels are already low. In the CRPC or mCRPC stage the mortality rate will increase higher (4).

Many factors have been identified from several existing studies to predict the occurrence of CRPC or mCRPC in prostate cancer patients who have received primary ADT. With differences in disease patterns and patterns of prostate cancer management in Indonesia, this study is the first study in Indonesia to identify factors that affect the occurrence of CRPC or mCRPC, with the hope that when these factors are known it is possible for more aggressive management to limit the occurrence of CRPC or mCRPC.

\section{MATERIALS AND METHODS}

This is a retrospective study in 205 prostate cancer patients with CRPC or mCRPC who came to the urology clinic at Saiful Anwar Malang Hospital from January 2013 to December 2018. The diagnosis of CRPC or mCRPC is based on the 2018 EAU guidelines criteria on CRPC / mCRPC where testosterone levels are $<50 \%$ / dL, with an increase in PSA of more than $50 \%$ nadir or an increase in PSA of more than $2 \mathrm{ng} / \mathrm{dL}$ in 3 measurements in a row with 1 week apart or 2 or more new metastasis in the bone or new metastases in the visceral organ. Diagnosis of prostate cancer is established both from transrectal biopsy and from the results of trans-urethral resection of prostate (TURP),

Published By:

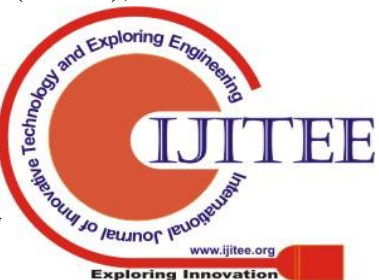


the clinical stage is based on clinical examination (digital rectal examination), bone survey, plain chest photo and abdominal ultrasound. All patients received primary ADT continuously in the form of bilateral orchidectomy, androgen blockage monotherapy in the form of LHRH agonist or complete androgen blockage in the form of LHRH agonist and antiandrogen.

The recorded parameters from the patient's medical record were age when the patient was diagnosed with prostate cancer, PSA levels before ADT, Gleason score, prostate cancer stage, type of ADT (surgery or medication), nadir PSA which was the lowest PSA level experienced by patients, the time between ADT and achieving PSA nadir in months, testosterone after ADT is examined 1 week after orchiectomy or 6 weeks after administration of ADT in the form of drugs, and time between ADT administration to occurrence CRPC or mCRPC.

Based on the time of CRPC or MCRPC, the patients were divided into group one and two, group one consist of CRPC with less than 2 years after ADT and group two, with the time of CRPC more than 2 years after the primary ADT. All recorded data will be compared between the two groups. To assess the relative risk factors in prostate cancer patients who had CRPC or mCRPC, univariate and multivariate analyzes including age ( $<60$ years vs. $\geq, 60$ years), PSA levels before ADT (<20ng / dL vs. $\geq$, 20ng / dL), Gleason score ( $<7$ vs $\geq$, $7)$, stage of prostate cancer (local vs. metastasi), nadir PSA ( $<4 \mathrm{ng} / \mathrm{dL}$ vs $\geq 4 \mathrm{ng} / \mathrm{dL}$ ), time between ADT and nadir PSA ( $<6$ months vs $\geq, 6$ months) and testosterone after ADT (<20ng / dL vs. $\geq 20 \mathrm{ng} / \mathrm{dL}$ ).

Statistical analysis using SPSS 20 for Macintosh and statistically significant if $\mathrm{p}<0.05$. To assess the differences in each group, the Mann Whitney test was used and to see independent factors influencing the occurrence of CRPC, the Cox proportional hazards regression model was used, only variables that were significant in the univariate analysis $(\mathrm{P}$ $<0.005$ ) were continued in multivariate analysis to see the most significant factors predict the occurrence of CRPC or mCRPC. This research was approved by the ethics commission at Saiful Anwar Hospital in Malang.

\section{RESULTS AND DISCUSSION}

The basic data character of all patients can be seen in table 1 , where the average age of patients is $67,53 \pm 6.86$ years with an average PSA level when diagnosed with prostate cancer is $674.87 \pm 1405.80$. A total of 189 (46.7\%) patients with prostate cancer who has underwent metastasis. The most widely used type of ADT is orchiectomy performed in $297(73.3 \%)$ patients. The highest Gleason score was 7 in 189 (46.7\% 0 patients. The average testosterone level after ADT was $19.04 \pm 13.7 \mathrm{ng} / \mathrm{dL}$ with a nadir PSA level of $16.94 \pm 19.9 \mathrm{ng} / \mathrm{dL}$ The average time needed to reached nadir PSA was $6.58 \pm 2.17$ months with the average time of CRPC occurrence was $24.7 \pm 9.74$ months.

Comparison between groups with a period of occurrence of CRPC less than 24 months and a period of CRPC after 24 months are shown in table 2 , where there were no significant differences in age and ADT type in the two groups (p 0.091 and p 0.065). The initial PSA, Gleason score, prostate cancer stage, testosterone levels after ADT, nadir PSA and time to reach nadir PSA between groups with a CRPC less than 24 months and a group with more than 24 months of CRPC were significantly different. The initial PSA, nadir PSA and testosterone levels after ADT were significantly higher in the group with a CRPC less than 24 months (p 0,000). It was also found that the time to reach a nadir PSA was significantly shorter in the group with a CRPC less than 24 months (p 0,000).

Table 1. Characteristics of patient baseline data

\begin{tabular}{|l|l|l|}
\hline \multicolumn{1}{|c|}{ Variable } & \multicolumn{1}{|c|}{ Mean } & \multicolumn{1}{c|}{ SD } \\
\hline Age (years) & 67,53 & 6,86 \\
\hline Initial PSA (ng/mL) & 674,87 & 1405,80 \\
\hline Gleason score (n) & $54(13 \%)$ & \\
$\mathbf{5}$ & $63(15,6 \%)$ & \\
$\mathbf{6}$ & $189(46,7 \%)$ & \\
$\mathbf{7}$ & $36(8,9 \%)$ & \\
$\mathbf{8}$ & $36(8,9 \%)$ & \\
$\mathbf{9}$ & $27(6,7 \%)$ & \\
$\mathbf{1 0}$ & $216(53,3 \%)$ & \\
\hline Stage (n) & $189(46,7 \%)$ & \\
Locally & & - \\
Locally advance + metastatic & $297(73,3 \%)$ & \\
\hline Type of ADT (n) & $108(26,7 \%)$ & \\
Surgical & 19,04 & 13,7 \\
Medical & & \\
\hline Testosterone level after ADT \\
(ng/dL) & 16,94 & 19,90 \\
\hline PSA nadir (ng/dL) & 6,58 & 2,17 \\
\hline Time to Nadir PSA (month) & 24,7 & 9,74 \\
\hline Time to CRPC (month) & & \\
\hline
\end{tabular}

In univariate analysis it was found that baseline PSA was> 20ng / dL (HR 1,562, p 0,000), Gleason score> 7 (HR 1,427, p 0,001), stage of cancer with metastasis (HR 2,025, p 0,000) testosterone after ADT> 20\% / dL (HR 5,090, p 0,000), nadir PSA> 4ng / dL (HR 2,314, p 0,000) and the time to reach a nadir PSA <6 months (HR 2,103, p 0,000) was a factor influencing the occurrence of CRPC progression shorter than 24 months. In multivariate analysis it was found

that the stage of cancer with metastasis (HR 1,616, p $0,048)$ testosterone after ADT with levels> 20ng / dL (HR 4,638, p 0,000), nadir PSA> 4ng / dL (HR 1,716, p 0,023) and time to reach Nadir PSA <6 months (HR 1.596, p 0.004) was a significant independent factor for CRPC occurrence less than 24 months can be seen in (Table 3 ).

\section{DISCUSSION}

Prostate cancer remains the second most diagnosed cancer in men (1). The frequency of autopsied prostate cancer is approximately the same in the whole world (1). The prevalence of prostate cancer at $<30$ years of age is $5 \%$, then increases with an odds ratio of 1.7 per decade, to a prevalence of $59 \%(48-71 \%)$ at age 79 years (2). 
Since it was discovered that prostate cancer is a type of androgen-sensitive cancer (testosterone), the use of anti-androgens or ADT is one of the main treatments in prostate cancer to date (5). The use of ADT will not stop the progression of cancer in patients who diagnosed prostate cancer because the cancer itself will evolve, so that in time the cancer is no longer dependent on androgens or testosterone. In this condition even though androgen production has been suppressed at the nadir, cancer will continue to grow and cause worse clinical progression. This condition is called CRPC. This condition of CRPC causes prostate cancer mortality to be even higher (4).

Table 2. Differences in variables between groups with a period of occurrence of CRPC less than 24 months and CRPC period after 24 months

\begin{tabular}{|l|l|l|l|}
\hline \multirow{2}{*}{ Variable } & \multicolumn{2}{c|}{ Time to CRPC } & \multirow{2}{*}{ p } \\
\cline { 2 - 3 } & $\langle 24$ months & $>24$ months & 0,091 \\
\hline Age (years) & $68,29 \pm 6,79$ & $66,88 \pm 6,87$ & 0,000 \\
\hline Initial PSA (ng/mL) & $1411,14 \pm 1795,67$ & $30,63 \pm 24,09$ & \\
\hline Gleason score (n) & & 36 & \\
$\mathbf{5}$ & 18 & 36 & \\
$\mathbf{6}$ & 0 & 63 & \\
$\mathbf{7}$ & 81 & 108 & \\
$\mathbf{8}$ & 36 & 0 & \\
$\mathbf{9}$ & 27 & 9 & 0,000 \\
$\mathbf{1 0}$ & 27 & 0 & \\
\hline Stage (n) & & & 0,065 \\
Locally & 30 & 177 & \\
Locally advance + metastatic & 150 & 39 & \\
\hline Type of ADT (n) & & & 171 \\
Surgical & 126 & 45 & 0,000 \\
Medical & 63 & $8,54 \pm 4,88$ & 0,000 \\
\hline Testosterone level after ADT (ng/dL) & $31,05 \pm 10,24$ & $2,72 \pm 2,90$ & 0,000 \\
\hline PSA nadir (ng/dL) & $33,20 \pm 18,54$ & $8,25 \pm 1,61$ & \\
\hline Time to Nadir PSA (month) & $4,67 \pm 0,56$ & & \\
\hline
\end{tabular}

Tabel 3. Estimated Hazard Ratio and Confidence intervals from proportional hazards modeling to progress into CRPC of less than 24 months

\begin{tabular}{|l|l|l|l|l|l|l|}
\hline \multirow{2}{*}{ Variable } & \multicolumn{2}{l}{ Univariate Analysis } & \multicolumn{3}{l|}{ Multivariate Analysis } \\
\cline { 2 - 7 } & $\mathrm{p}$ & $\mathrm{HR}$ & $\mathrm{CI}(95 \%)$ & $\mathrm{p}$ & HR & CI (95\%) \\
\hline Age < 60 years & 0,128 & 0,766 & $0,543-1,080$ & - & - & - \\
\hline PSA > 20 ng/dL & 0,000 & 1,562 & $1,261-1,935$ & 0,520 & 0,904 & $0,664-1,230$ \\
\hline GS >7 & 0,001 & 1,427 & $1,155-1,763$ & 0,801 & 1,038 & $0,777-1,387$ \\
\hline Stage (locally advance and metastatic) & 0,000 & 2,025 & $1,643-2,494$ & 0,048 & 1,616 & $1,098-1,769$ \\
\hline Testosterone after ADT > 20 ng/dL & 0,000 & 5,090 & $3,916-6,615$ & 0,000 & 4,638 & $3,470-6,199$ \\
\hline Nadir PSA >4 ng/dL & 0,000 & 2,314 & $1,865-2,872$ & 0,023 & 1,716 & $1,210-1,936$ \\
\hline Time to Nadir PSA <6 months & 0,000 & 2,103 & $1,702-2,599$ & 0,004 & 1,596 & $1,005-1,545$ \\
\hline
\end{tabular}

There are many factors that can be used to predict the progression of prostate cancer that has received ADT to become CRPC. The most basic thing is the PSA profile, both the initial PSA, the nadir PSA after ADT, the PSA doubling time and the time required to become a nadir PSA. PSA itself is currently the main marker for prostate cancer, both for diagnosis, stage classification, evaluation of treatment success and for assessing the progression of prostate cancer. PSA monitoring before and after ADT is something useful to determine the treatment response of the patient. PSA concentration decreases $60-80 \%$ after ADT and lasts for 3-6 months. Significant increase in PSA indicates a worsening progression of prostate cancer (6-9). From this study almost all patients responded to ADt where there was a decrease in PSA from the initial average of $674.87 \mathrm{ng} / \mathrm{dL}$, to 16, $94 \mathrm{ng} /$ $\mathrm{dL}$ or about $97 \%$. And the average time needed to re-increase the PSA is 24 months. Some studies say that early PSA levels before ADT therapy has no effect on prostate cancer progression, but several other studies say that the initial PSA have no effect. Nadir PSA, time taken to be a nadir PSA, gleason score and clinical stage are important prognostic factors to see the efficacy of ADT therapy. Nadir PSA ( $<4 n g$ / $\mathrm{dL}$ ) is more useful to see when the progression of the disease is compared to the gleason score (10-15).

Nadir PSA is one of the PSA kinetic profiles that can be used as a benchmark to see the progression of prostate cancer. The lower the minimum PSA, the more likely the progress to become a CRPC will be. When patients with prostate cancer after ADT cannot reach a nadir PSA < 1ng / $\mathrm{dL}$, the likelihood progression to CRPC is also faster $(11,14$, 16-19). From this study it was found that nadir PSA was one of the independent factors to see the progression of CRPC and when patients were unable to reach a nadir PSA $<4 \mathrm{ng} /$ $\mathrm{dL}$, 
the progression to CRPC in less than 24 months rise 1.7 times compared to patients who were able reach nadir PSA $<4 \mathrm{ng} / \mathrm{dL}$.

Another factor that is used as a parameter to see the progression of prostate cancer is the time needed to reach the nadir PSA was PSA kinetic. Many studies show that as soon as the time needed to become a nadir PSA, the faster the progression of prostate cancer to become CRPC (20), in this study it was found that the time needed to become a nadir PSA is also one of the independent factors to see the progression of prostate cancer, where the time to reach a minimum PSA of $<6$ months, the probability of progression of patients to CRPC less than 24 months is 1,596 times higher compared to patients who have time to reach a nadir PSA of more than 6 months. The exact mechanism for explaining this phenomenon still remain unclear. Research from Sasaki T and Asugimura in 2018 explained the possible basic mechanism causing this phenomenon. It is believed that around the prostate cancer stroma there were fibroblasts called cancer associated fibroblasts (CAFs), in the circumstances after ADT where drastic androgen / testosterone is obtained which will cause damage to prostate cancer cells. The faster the decrease in testosterone levels, the more cancer cells affected, and more and more CAFs are activated. Activation of CAFs alone will increase the proliferation of prostate cancer that is independent of androgens so CRPC will appear (21). This postulate is supported by several clinical studies when combining nadir PSA, the Gleason score and stage of cancer will be able to predict the progression of prostate cancer. Nadir PSA $<0.2$ $\mathrm{ng} / \mathrm{dL}$ and time to reach PSA nadir $>9$ months is the initial predictor to see the progression of bone metastatic prostate cancer (22).

Testosterone levels after ADT is also one of the parameters used to see the progression of prostate cancer after ADT. Research from Wang, et al., 2017 shows that testosterone $<25 \mathrm{ng} / \mathrm{dL}$ after ADT is a good predictor factor for preventing progression of prostate cancer faster than testosterone levels <50ng / dL after ADT (23). Another study from Shiota et al. In 2015 even stated that testosterone levels before and after therapy was predictor factor for the progression of prostate cancer, the higher testosterone levels before and after ADT, the progression of prostate cancer will occur faster (24). In this study, testosterone levels before therapy was not measured so that they were not included in the study variables, but testosterone levels after ADT was independent factor to see how fast prostate cancer progression became CRPC. Patients with testosterone> 20ng / dL after ADT have a 4.63-fold chance of shorter than 24 months to CRPC compared to patients with testosterone levels <20ng / dL after ADT.

The disadvantages of this study were that samples was taken from retrospective data where some supporting data could not be included such as testosterone before ADT, lactate dehydrogenase (LDH), alkaline phosphatase (ALP), albumin, because there are npt in our management protocols of prostate cancer patients. Staging of prostate cancer itself is limited to physical examination, ultrasound, bone survey and

plain chest radiograph without using MRI or CT scans because of the limited facilities. Another limitation was the possibility of variations in the reading of the Gleason score because it was examined by single doctor.

\section{CONCLUSION}

Cancer stage, testosterone levels after ADT, nadir PSA and time needed to reach nadir PSA were independent factors for CRPC in patients with prostate cancer who had primary ADT.

\section{REFERENCES}

1. Mottet, N., Bellmunt, J., Bolla, M., Briers, E., Cumberbatch, M.G., De Santis, M., Fossati, N., Gross, T., Henry, A.M., Joniau, S. and Lam, T.B., 2018. EAU-ESTRO-SIOG guidelines on prostate cancer. European urology, 71(4), pp.618-629.

2. Bell, K.J., et al. Prevalence of incidental prostate cancer: A systematic review of autopsy studies. Int J Cancer, 2015. 137: 1749.

3. KPKN. Komite Penanggulangan Kanker Nasional. 2017. Panduan Penatalaksanaan Kanker Prostat. Kementerian Kesehatan Republik Indonesia.

4. NIH (National Cancer Institure. 2018. Cancer Stat Facts: Prostate Cancer. https://seer.cancer.gov/statfacts/html/prost.html (Accessed on December 25, 2018)

5. Kim M, Lee J, Jeong CW, et al. 2015. Prostate-specific antigen kinetic profiles during androgen deprivation therapy as prognostic factors in castration-resistant prostate cancer. Urol Oncol. 2015 May;33(5):203.e1-9

6. Arai Y, Yoshiki T, Yoshida O: Prognostic significance of prostate specific antigen in endocrine treatment for prostatic cancer. J Urol 1990, 144:1415-1419.

7. Matzkin H, Eber P, Todd B, van der Zwaag R, Soloway MS: Prognostic significance of changes in prostate-specific markers after endocrine treatment of stage D2 prostatic cancer. Cancer 1992, 70:2302-2309.

8. Newling DW, Denis L, Vermeylen K: Orchiectomy versus goserelin and flutamide in the treatment of newly diagnosed metastatic prostate cancer. Analysis of the criteria of evaluation used in the European Organization for Research and Treatment of Cancer-Genitourinary Group Study 30853. Cancer 1993, 72:3793-3798.

9. Scher HI, Halabi S, Tannock I, Morris M, Stemberg CN, Carducci MA, Eisenberger MA, Higano C, Bubley GJ, Dreicer R, Petrylak D, Kantoff P, Basch E, Kelly WK, Figg WD, Small EJ, Beer TM, Wilding G, Martin A, Hussain M: Prostate Cancer Clinical Trials Working Group: Design and end points of clinical trials for patients with progressive prostate cancer and castrate levels of testosterone: recommendations of the Prostate Cancer Clinical Trials Working Group. J Clin Oncol 2008, 26:1148-1159.

10. Miller JI, Ahmann FR, Drach GW, Emerson SS, Bottaccini MR: The clinical usefulness of serum prostate specific antigen after hormonal therapy of metastatic prostate cancer. J Urol 1992, 147:956-961

11. Benaim EA, Pace CM, Lam PM, Roehrborn CG: Nadir prostate-specific anti- gen as a predictor of progression to androgen-independent prostate cancer. Urology 2002, 59:73-78.

12. Cooper EH: Prostate specific antigen in diagnosis, staging, and follow-up of prostate cancer. Prostate Suppl 1992, 4:125-128.

13. Dijkman GA, Janknegt RA, De Reijke TM, Debruyne FM: Long-term efficacy and safety of nilutamide plus castration in advanced prostate cancer, and the significance of early prostate specific antigen normalization. International Anandron Study Group. J Urol 1997, 158:160-163

14. Morote J, Trilla E, Esquena S, Abascal JM, Reventos J: Nadir prostate-specific antigen best predicts the progression to androgen-independent prostate cancer. Int J Cancer 2004, 108:877-881.

15. Kiper A, Yigitbasi O, Imamoglu A, Tuygun C, Turan C: The prognostic importance of prostate specific antigen in the monitorisation of patients undergoing maximum androgen blockade for metastatic prostate cancer. Int Urol Nephrol 2006, 38:571-576.

16. Choueiri TK, Xie W, D'Amico AV, Ross EW, Hu JC, Pomerantz M, Regan MM, Taplin ME, Kantoff PW, Sartor O, Oh WK: Time to prostate-specific antigen nadir independently predicts overall survival in patients who have metastatic hormone-sensitive prostate cancer treated with androgen-deprivation therapy. Cancer 2009, 115:981-987. 
17. Kwak C, Jeong SJ, Park MS, Lee E, Lee SE: Prognostic significance of the nadir prostate specific antigen level after hormone therapy for prostate cancer. J Urol 2002, 168:995-1000.

18. Park SC, Rim JS, Choi HY, Kim CS, Hong SJ, Kim WJ, Lee SE, Song JM, Yoon JH: Failing to achieve a nadir prostate-specific antigen after combined androgen blockade: predictive factors. Int J Urol 2009, 16:670-675.

19. Sim HG, Lau WK, Cheng CW: Predictors of androgen independence in metastatic prostate cancer. BJU Int 2004, 93:1221-1224.

20. Atsushi Tomioka, Nobumichi Tanaka, Motokiyo Yoshikawa, Makito Miyake, Satoshi Anai, Yoshitomo Chihara, Eijiro Okajima, Akihide Hirayama, Yoshihiko Hirao and Kiyohide Fujimoto. Nadir PSA level and time to nadir PSA are prognostic factors in patients with metastatic prostate cancer. BMC Urology 2014, 14:33

21. Sasaki T, Sugimura Y. The Importance of Time to Prostate-Specific Antigen (PSA) Nadir after Primary Androgen Deprivation Therapy in Hormone-Naïve Prostate Cancer Patients. J. Clin. Med. 2018, 7(12), 565

22. Sasaki T, Ohnishi T, Hoshina A: Nadir PSA level and time to PSA nadir following primary androgen deprivation therapy are the early survival predictors for prostate cancer patients with bone metastasis. Prostate Cancer Prostatic dis 2011, 14:248-252.

23. Wang Y, Dai B, Ye DW. Serum testosterone level predicts the effective time of androgen deprivation therapy in metastatic prostate cancer patients. Asian J Androl. 2017 Mar-Apr; 19(2): 178-183

24. Masaki Shiota, Ario Takeuchi, Masaaki Sugimoto, Eiji Kashiwagi, Takashi Dejima, Keijiro Kiyoshima, Junichi Inokuchi, Katsunori Tatsugami and Akira Yokomizo . Prognostic Impact of Serum Testosterone and Body Mass Index Before Androgen-deprivation Therapy in Metastatic Prostate Cancer. Anticancer Research 35: 6925-6932 (2015)

\section{AUTHORS PROFILE}

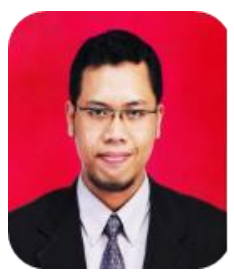

Dr. Taufiq Nur Budaya Sp. U has completed his urologist degree from Airlangga University, $\mathrm{He}$ is currently working as urology staff at Urology Department, Brawijaya University, Malang. He has released 2 books about $\mathrm{BPH}$ and Malignancy in Urinary Tract.

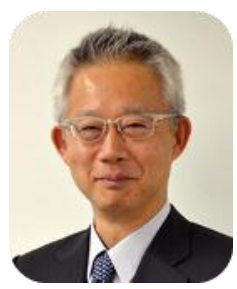

Horie Shigeo, M.D., Ph.D. has completed his urologist from The University of Tokyo. He is currently as a Professor at Urology Department, Faculty of Medicine, Juntendo University. He has published 100 original articles in various national and international journal. He has released 1 book. 